\title{
Big Data Service Architecture for E-portal
}

\author{
Vaishnavi Chillal $^{1}$, Nikita Dalvi ${ }^{2}$, Bhagyashree Nagapure ${ }^{3}$, Shamla Naidu ${ }^{4}$, Prof. Rajesh Bharati ${ }^{5}$ \\ Dept of Computer Engineering, Dr. D. Y. Patil Institute of Technology, Savitribai Phule Pune University ${ }^{1,2,3,4,5}$
}

\begin{abstract}
The system is being designed in such a way that the E-banking portal is being used by the user to do the multiple transactions on the system, as the bank application works. Tha Data being collated is being stored onto Hadoop as storage as well as for Analytics using MapReduce. Huge data is being collated all the predictive results has been acquired using hadoop. In this context, the ability to apply promising state-of-the-art big data technologies to extract the maximum value from the vast amounts of the data generated is generating a lot of interest in the financial services industry. The main motivation of the system is to permit advanced trace information analysis, the unreal cloud design ought to accommodate the access to capricious sets of the trace information.
\end{abstract}

Keywords: Hadoop, MapReduce, Big Data.

\section{INTRODUCTION}

\section{BigData and Hadoop:}

Massive records is an evolving time period that describes any voluminous quantity of structured, semi structured and unstructured records that has the ability to be mined for statistics.

Hadoop is a open-source software framework that supports facts-extensive dispensed applications, licensed below the apache v2 license.

\section{Benefits of Hadoop:}

1. Abstract and facilitate the storage and processing of large and/or rapidly growing data sets

2. Structured and non-structured data

3. Simple programming models

4. High scalability and availability

5. Use commodity (cheap!) hardware with little redundancy and Fault-tolerance

6. More computation rather than data

\section{MapReduce:}

MapReduce is a processing method and a application version for distributed computing based totally on java. It includes the two foremost obligations namely map and decrease. The lessen assignment is always carried out after the map project. Underneath the mapreduce model, the facts processing primitives are called mappers and reducers.

\section{Mapper:}

- Given (filename, a few text) and "sample", if "textual content" suits "sample" output (filename, _)

\section{Reducer:}

- Identity feature the fundamental benefit of this technique is that it is easy to scale records processing over multiple computing nodes.

- the device is designed in any such way that the e-banking portal is being used by the user to do the multiple transactions at the gadget, because the financial institution utility works.

- in banking programs, there are large quantity of transactions are done by customers daily, to save those statistics machine wishes large records storage. therefore device proposes an architecture which is using cloud garage for analyzing huge tracking records.

- the hadoop machine provides a value-effective manner to keep big volumes of dependent and unstructured facts in one vicinity for deep evaluation.

- cloud server architecture affords velocity, safety, scalability, reliability and availability to the gadget. consequently the usage of cloud structure machine analyse information with protection and with fast speed which may be very efficient for banking transactions.

\section{OBJECTIVE}

1. The main motivation of the system is to permit advanced trace information analysis, the unreal cloud design ought to accommodate the access to capricious sets of the trace information. Additionally, the cloud design desires a web-based interface for building structured queries of knowledge and displaying the results. 
2. The system is being designed in an exceedingly such some way that the E-banking portal is getting used by the user to try to the multiple dealing on the system, because the bank application works. Huge information is being collated all the prophetical results has been non inheritable exploitation hadoop. The Hadoop system provides an economical thanks to store giant volumes of structured and unstructured information in one place for deep analysis.

\section{LITERATURE SURVEY}

\section{Cloud server architecture for analyzing big monitoring data}

Cloud tracking is of a source of large information which are constantly created from strains of infrastructures, systems, and packages. Analysis of monitoring information can provide insights of the gadget's workload and usage sample and guarantees workloads are working at superior stages. The analysis process entails records query and extraction, statistics analysis, and end result visualization. For the reason that quantity of monitoring facts is big, those operations require a scalable and dependable architecture to extract, mixture, and examine facts in an arbitrary variety of granularity. Ultimately, the results of analysis come to be the understanding of the gadget and have to be shared and communicated. The paper affords cloud service structure that explores a search cluster for data indexing and query. The authors increase relaxation APIS that the statistics can be accessed by way of special analysis modules.

2 . Big data analytics using Hadoop

The volume and the heterogeneity of records with the rate it's miles generated, makes it hard for the existing computing infrastructure to control massive statistics. Conventional information control, warehousing and evaluation structures fall brief of tools to investigate this information. Due to its particular nature of huge information, it's miles saved in disbursed document gadget architectures. Hadoop and HDFS with the aid of apache is broadly used for storing and managing large records. Reading massive facts is a tough undertaking as it involves huge allotted document structures which must be fault tolerant, bendy and scalable. Map reduce is extensively been used for the efficient evaluation of huge statistics. Traditional DBMS strategies like joins and indexing and different strategies like graph seek is used for category and clustering of big data. Those techniques are being adopted for use in map reduce.

3. A Cloud-Based Network Architecture for Big Data Services

Massive facts offerings are predicted to convey large capability for commerce, enterprise and studies. In fact, collection of state-of-the-art approaches are concerned in big records services. but, there exists a structural gap, that's maintaining lower back the improvement of huge statistics offerings. Here four-layer cloud-based totally network architecture become proposed to assist massive records offerings. The network structure presents a systematic and efficient approach for huge records get admission to, storage and retrieval. Specifically, the techniques for supporting huge information services are categorized into information transfer, statistics collection, facts processing and statistics retrieval. Furthermore, a cloud-primarily based structure turned into provided, followed through a closer have a look at the community structure for large statistics collection at tool degree.

4. Automatic Cloud Services Composition for Big Data Management

The exploitation of cloud infrastructure in huge information management is attractive due to charges reductions and potentiality of garage, community and computing sources. The cloud can always reduce the cost of analysis of facts from one of a kind sources, commencing analytics to large storages in a multi-cloud environment. Developing and executing this form of service could be very complicated when you consider that one-of-a-kind assets must be provisioned and coordinated depending on users' wishes. Orchestration is a strategy to this trouble, however it requires proper languages and methodologies for automatic composition and execution.

5. Building big data and analytics solution in the cloud

Cloud computing appears to be a super car for web hosting huge statistics workloads. However, working on massive facts within the cloud brings its own undertaking of reconciling contradictory layout concepts. Cloud computing is primarily based on the concepts of consolidation and resource pooling, but big statistics structures (including hadoop) are built on the shared nothing principle, in which each node is unbiased and self-enough. an answer structure that may allow these collectively exclusive ideas to coexist is required to absolutely make the most the pliancy and ease-of-use of cloud computing for huge data environments. Records regarding how big statistics affects cloud surroundings, purposeful and infrastructure architecture concerns for massive records and statistics analytics in the cloud and implementation styles to solve the maximum commonplace large information use cases.

\section{PROPOSED SYSTEM AND ARCHITECTURE}

The main intention behind the commencement of e-banking services is to provide the customers with an alternative that is more responsive and with less expensive options. The main objective of this project is to demonstrate by using Hadoop concepts, how data generated from YouTube can be mined and utilized to make targeted, real time and informed decisions. HDFS (Hadoop Distributed File System) is a primary Hadoop application and a user can directly interact with HDFS using various shell-like commands supported by Hadoop. This project uses SQL like queries that 
are later run on Big Data using Hadoop to extract the meaningful output which can be used by the management for analysis.
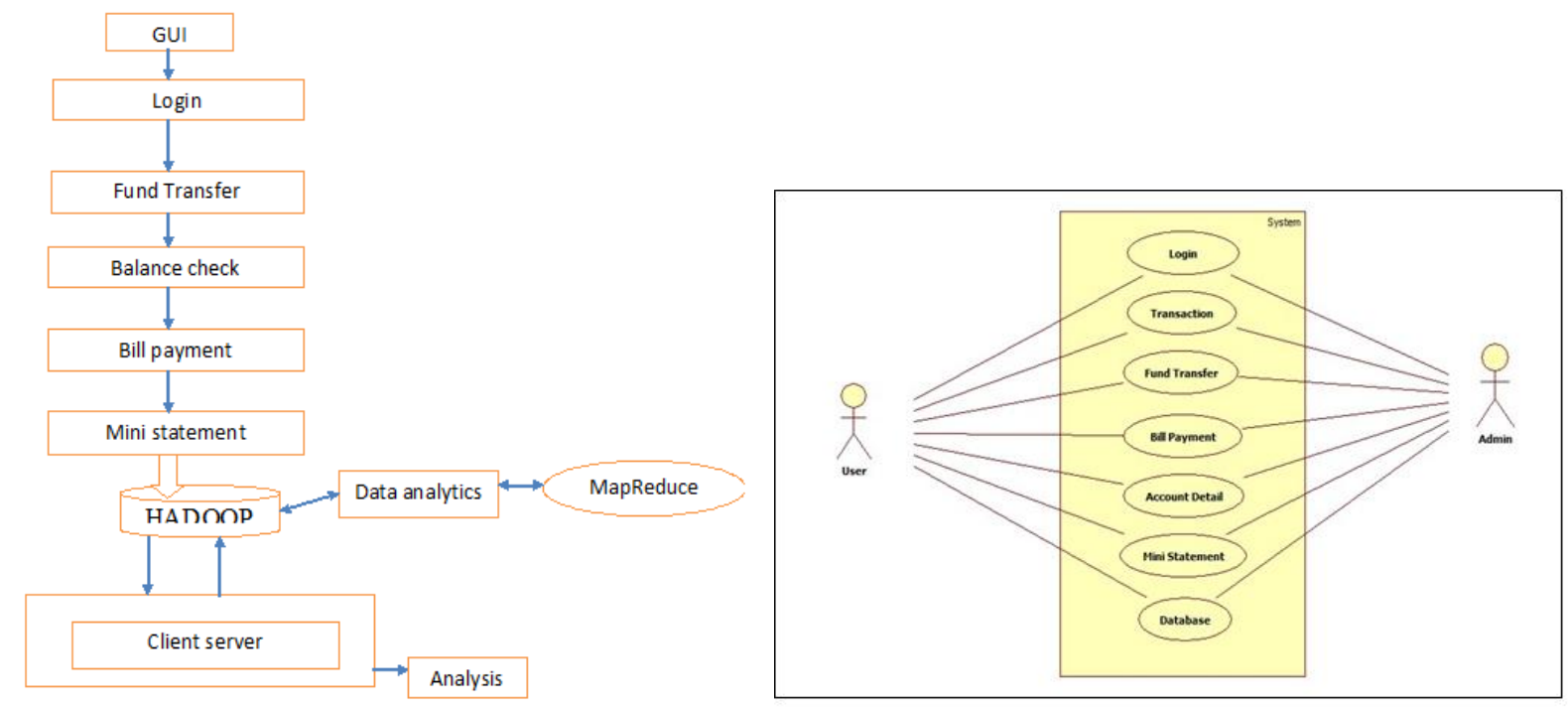

Fig. Proposed System

The system is being designed in such a way that the E-banking portal is being used by the user to do the multiple transactions on the system, as the bank application works. Tha Data being collated is being stored onto Hadoop as storage as well as for Analytics using MapReduce. Huge data is being collated all the predictive results has been acquired using hadoop. In this context, the ability to apply promising state-of-the-art big data technologies to extract the maximum value from the vast amounts of the data generated is generating a lot of interest in the financial services industry.

- $\quad$ Data Design

Database design is the process of producing a detailed data model of database. This data model contains all the needed logical and physical design choices and physical storage parameters needed to generate a design in a data definition language, which can then be used to create a database.

- Internal software data structure

When SQL returns the results of the query sent to it by JSP, the results of the query will be passed back to JSP using the built in data structures through the SERVLET.

- $\quad$ Temporary data structure

We will be using a cookie saved on the users machine to temporarily store the users query entry. This is so the user can go back to the query page and easily modify their last query to refine or widen their search as needed

\section{ALGORITHMIC PSEUDO CODE}

We are going to use the AES (Advanced EncryptionStandard) algorithm. AES is based upon substitution-permutation network. It performs all the computations on bytes rather than bits. It is almost six times faster than triple DES. The replacement for DES was needed because its key size was too small. It is basically a key based algorithm. Key sizes accommodated are 128,192 or 256 bits.

1. I / P : A 4 word ( 16 b y te ) key

2. Cipher(bytein[16], byteout [ 16$]$, keyarrayroundkey [ $\mathrm{Nr}+1]$ )

3. begin

4. byte state[ $[16]$;

5. $\quad \mathrm{stat}$ e $=$ in ;

6. AddRoundKey ( s t a te, roundke y [0]);

7. for $\mathrm{i}=1 \mathrm{t}$ o $\mathrm{Nr}-1 \mathrm{~s}$ te $\mathrm{psize} 1$ do

8. $\quad$ SubBytes ( s t a te );

9. $\quad$ ShiftRows ( s t a te );

10. MixColumns ( s t a te ); AddRoundKey ( s t a t e, r o und ke y [i ] );

11. end for

12. SubBytes ( s t a te ); 
13. ShiftRows ( s t a t e );

14. AddRoundKey ( $\mathrm{s} \mathrm{t}$ a t e, roundke y [ $\mathrm{Nr}]$ );

15. end

\section{CONCLUSION}

Hadoop(MapReduce) is one of the capable systems that empower simple advancement on information serious application. It goal is help constructing a supplication with high versatility with a large number of machines. We can see Hadoop is extremely reasonable to information concentrated foundation application and flawless fit to our projects requirements.

In this framework, we proposed design for distributed storage which is exceptionally useful for checking enormous information. The framework is outlined in a such a path, to the point that the E-banking entrance is being utilized by the client to do the various exchange on the framework, as the bank application works at the back end information will be gathered onto hadoop and the tremendous information will be put away on to the server and the entire information will be put away on to the hadoop as a capacity reason and hence do the various analytics..

\section{ACKNOWLEDGMENT}

We would like to express my deepest appreciation to all who provided me the possibility to complete this paper. A special gratitude we give to our project guide Prof Rajesh Bharati, whose contribution in stimulating suggestions and encouragement, helped me in writing this paper.

\section{REFERENCES}

(1) A cloud server architecture for analyzing big monitoring data; Samneet Singh and Yan Liu et.al.

(2) Big data analytics using Hadoop; Bijesh Dhyani, Anurag Barthwal et.al.

(3) Cloud-Based Network Architecture for Big Data Services; Ming Zhao, Arun Kumar et.al

(4) Automatic Cloud Services Composition for Big Data Management; Flora Amato, Francesco Moscato et.al

(5) Building big data and analytics solution in the cloud; Wei-Dong (Jackie) Zhu Manav Gupta et.al

(6) Big Data- solutions for RDBMS problems-A Survey; S.Vikram Phaneendra E.Madhusudhan Reddy.;In 12th IEEE/IFIP Network Operations Management Symposium (NOMS 2010) (Osaka, Japan, Apr 1923 2013).

(7) Analysis of Bidgata using Apache Hadoop and Map Reduce; Mrigank Mridul, Akashdeep Khajuria, Snehasish Dutta, Kumar N.;Volume 4, Issue 5, May 201427

(8) Shared disk big data analytics with Apache Hadoop; Mukherjee, A.; Datta, J.Jorapur, R.; Singhvi, R.; Haloi, S.; Akram;2012, 18-22

(9) Big data integration; Dong, X.L.; Srivastava, D. Data Engineering (ICDE);IEEE International Conference on ,29(2013) 1245124

(10) Implementation of a Cloud in Banking Sector; Chitrali Agre;International Journal of Computer Science and Information Technology Research ISSN 2348-120X (online) Vol. 3, Issue 2, pp: (1168-1174), Month: April - June 2015 